\title{
Mineração
}

\section{Reconciliação pró-ativa em empreendimentos mineiros}

\section{(Proactive reconciliation in mining industry)}

\author{
Ana Carolina Chieregati \\ Professora, Dra., Departamento de Engenharia de Minas e de Petróleo, Escola Politécnica da USP \\ E-mail: ana.chieregati@poli.usp.br \\ Homero Delboni Jr. \\ Professor, Dr., Departamento de Engenharia de Minas e de Petróleo, Escola Politécnica da USP \\ E-mail:hdelboni@usp.br \\ João Felipe Coimbra Leite Costa \\ Professor, Dr., Departamento de Engenharia de Minas, Universidade Federal do Rio Grande do Sul \\ E-mail: jfelipe@ufrgs.br \\ Fernanda Bastos Carneiro \\ Engenheira de Minas, Rio Paracatu Mineração, Kinross. \\ E-mail:fernanda.carneiro@kinross.com
}

\section{Resumo}

A prática comum de reconciliação baseia-se na definição do mine call factor (MCF) e sua aplicação às estimativas dos modelos de longo e de curto prazo. O MCF expressa a diferença entre a produção prevista pelos modelos e a produção registrada na usina e, portanto, sua aplicação permite corrigir futuras estimativas. Esta é uma prática de reconciliação reativa. Entretanto a aplicação desses fatores às estimativas dos modelos pode mascarar as causas dos erros responsáveis pelas discrepâncias observadas. As causas reais de qualquer variância só podem ser identificadas analisando-se as informações referentes a cada variância e, em seguida, modificando metodologias e processos. Este é o conceito de prognosticação, ou reconciliação pró-ativa, um processo iterativo de recalibração constante dos dados de entrada e dos cálculos. Portanto a prognosticação permite uma correção das metodologias de coleta de dados, e não, simplesmente, uma correção das estimativas dos modelos. O presente trabalho analisa as práticas de reconciliação realizadas em uma mina de ouro no Brasil e sugere um novo protocolo de amostragem, com base nos conceitos de prognosticação.

Palavras-chave: Reconciliação, prognosticação, amostragem.

\begin{abstract}
The common practice of reconciliation is based on definition of the mine call factor (MCF) and its application to resource or grade control estimates. The $M C F$ expresses the difference, a ratio or percentage, between the predicted grade and the grade reported by the plant. Therefore, its application allows to correct future estimates. This practice is named reactive reconciliation. However, the use of generic factors that are applied across differing time scales and material types often disguises the causes of the error responsible for the discrepancy. The root causes of any given variance can only be identified by analyzing the information behind any variance and, then, making changes to methodologies and processes. This practice is named prognostication, or proactive reconciliation, an iterative process resulting in constant recalibration of the inputs and the calculations. The prognostication allows personnel to adjust processes so that results align within acceptable tolerance ranges, and not only to correct model estimates. This study analyses the reconciliation practices performed at a gold mine in Brazil and suggests a new sampling protocol, based on prognostication concepts.
\end{abstract}

Keywords: Reconciliation, prognostication, sampling. 


\section{Introdução}

A reconciliação é uma atividade desenvolvida na maior parte das empresas de mineração e pode ser definida como a comparação entre uma estimativa e uma medição, ou seja, entre os teores de minério estimados pelos modelos da jazida e os teores produzidos na usina de beneficiamento. Grandes discrepâncias entre esses valores são um problema comum em diversas minas de ouro e de metal básico no mundo, fazendo-se necessária a adoção de estratégias que minimizem esse problema.

Historicamente, a reconciliação tem sido realizada de maneira reativa, i.e., comparando-se os valores de produção com os valores estimados pelos modelos e aplicando-se fatores, tais como o mine call factor (MCF), a estimativas futuras, na tentativa de melhorar a previsão do desempenho de uma operação. Entretanto, conforme observou Morley (2003), esta não é melhor prática industrial de reconciliação. Uma prática correta de reconciliação deve ser realizada de maneira pró-ativa, i.e., identificando as causas das variâncias observadas e modificando as metodologias de coleta de dados e os processos. Só assim podem ser tomadas ações que reduzam a variância a um nível aceitável.

A prognosticação é uma alternativa à reconciliação e permite ajustar os processos de modo que os resultados estejam sempre dentro de limites aceitáveis. Os erros são analisados com a finalidade de se tomarem ações corretivas para assegurar que a diferença entre os valores estimados e os valores medidos seja mínima. Dessa maneira, as estimativas tornam-se previsões - ou prognósticos - e podem formar uma base para a tomada de decisões, assegurando que o que acontecerá no futuro corresponde ao que foi planejado no presente.

A reconciliação é vista como um teste de qualidade das estimativas dos modelos, porém, sem uma amostragem adequada, capaz de gerar dados confiáveis, qualquer análise perde o sentido. Um ato correto de amostragem requer que todas as partículas pertencentes a um determinado lote tenham a mesma chance de ser extraídas (François-Bongarçon \& Gy, 2002). Esse tipo de amostragem denomina-se amostragem nãoenviesada. Devido à falta de conhecimento dos fundamentos da teoria da amostragem, muitas empresas perdem milhões de dólares por ano com problemas de reconciliação. Estudos demonstram que mesmo pequenas melhorias na amostragem resultam em melhorias significativas nos resultados de uma operação.

$\mathrm{O}$ trabalho apresentado a seguir tem por objetivo desenvolver um sistema integrado de reconciliação pró-ativa, ou prognosticação, fundamentado no processo iterativo de análise de variâncias e correção de metodologias de coleta de amostras. Com base nesse sistema, são propostas alterações no protocolo de amostragem de um empreendimento mineiro, visando a diminuir os erros de amostragem, aumentar a confiabilidade nos dados e melhorar as estimativas dos modelos.

\section{Materiais e métodos}

Segundo Crawford (2004), a reconciliação não deve, simplesmente, avaliar os modelos de recursos e de controle de teor com base no produto final. Na prática, cada etapa da operação deve ser examinada seqüencialmente: do modelo de recursos para a mina, da mina para a usina (mine-to-mill) e da usina para a metalurgia ou produto final. O presente trabalho está focado na segunda etapa de reconciliação, ou seja, na etapa de comparação entre os teores da lavra e os teores alimentados na usina.

As estimativas para o planejamento de curto prazo são, em geral, baseadas em perfurações adicionais e em amostragens de frentes de lavra. Na maioria dos casos, utiliza-se amostragem do pó de perfuratriz proveniente dos furos de desmonte, o que apresenta duas vantagens principais: (a) o espaçamento entre os furos é pequeno, resultando em uma densidade de amostragem relativamente alta, e (b) já que os furos têm que ser perfurados de qualquer maneira, não há custo adicional de perfuração. $\mathrm{O}$ problema mais sério da amostragem de furos de desmonte é o enviesamento das amostras causado pela segregação de densidade e de tamanho das partículas. Uma das principais causas desse enviesamento é a perda de finos, o que pode levar a uma subestimativa ou a uma superestimativa do teor do minério (Snowden, 1993). O enviesamento da amostragem é, provavelmente, o erro mais difícil de se medir e, certamente, merece uma consideração especial. Segundo Gy (1998), "a heterogeneidade é vista como a única fonte de todos os erros de amostragem" e é a única condição na qual um conjunto de unidades pode ser observado na prática.

O planejamento de curto prazo anteriormente realizado na mina em estudo baseava-se na amostragem manual por pá. Porém esse tipo de amostragem não é correta, pois pressupõe que as partículas dispostas nas porções inalcançáveis pela pá apresentem as mesmas características de qualidade das partículas dispostas nas porções mais externas e superficiais da pilha. Assim, na amostragem manual por pá, além da variabilidade existente entre as partículas, os erros de amostragem são influenciados pela disposição das partículas no interior do lote. Em conseqüência, a precisão da amostragem manual não pode ser estimada, tornando-a não confiável (Grigorieffet al., 2002).

O procedimento experimental adotado objetivou minimizar os erros anteriormente descritos, desenvolvendo um amostrador que pudesse reduzir a perda de finos e aumentar a representatividade das amostras. A alternativa escolhida foi a utilização de um amostrador setorial estacionário, proposto por Pitard (1993), colocado ao redor do furo de desmonte. Esse tipo de amostrador minimiza os riscos de contaminação do material e os erros cometidos na amostragem manual. Ao amostrador proposto por Pitard, foi sugerida uma modificação visando a minimizar a perda de finos, um problema constante na amostragem de furos de desmonte. Essa modificação consistiu em adicionar uma cúpula semi- 
esférica ao amostrador, de modo que todo o material proveniente do furo pudesse ser coletado, diminuindo, assim, o enviesamento das amostras. A cúpula foi construída em material acrílico e possui a forma de uma semi-esfera, respeitando as condições de correção de extração da amostra. $\mathrm{O}$ amostrador proposto está ilustrado na Figura 1.

O amostrador setorial é encaixado à lança da perfuratriz e gera duas amostras, uma por recipiente, cada uma pesando, em média, $3 \mathrm{~kg}$. Os recipientes setoriais são dispostos em dois quadrantes do amostrador e suas arestas são alinhadas com o centro do furo. Cada recipiente coleta um setor da amostra, em forma de pizza, e o restante do material é descartado. Este amostrador é dimensionado de modo que a amostra coletada seja correta, seguindo a teoria de amostragem de Pierre Gy. O equipamento deve dar chances iguais de seleção para todas as partículas do lote e, também, deve amostrar todo o comprimento do furo. A Figura 2 mostra o amostrador encaixado à lança da perfuratriz.

A metodologia de amostragem foi baseada nos conceitos de reconciliação pró-ativa - ou prognosticação - de Morley, cujos passos tomados seqüencialmente seguiram um processo iterativo de alterações nos métodos de amostragem, visando à diminuição dos erros de estimativa e das variâncias dos erros de amostragem. Nesse processo, as alterações realizadas entre cada passo visaram a melhorar a qualidade das amostras, aumentando, portanto, sua representatividade.

O método de reconciliação consistiu na comparação entre os teores das amostras coletadas na usina (head samples) e os teores das amostras coletadas na mina (grade control samples). A amostragem na usina foi efetuada nos transportadores de correia que alimentam a moagem, a intervalos de tempo regulares, consistindo de material equivalente a $1 \mathrm{~m}$ de correia e pesando, em média, $50 \mathrm{~kg}$ cada. As amostras coletadas na mina foram provenientes dos furos de desmonte, utilizando o amostrador setorial descrito anteriormente.

O total de amostras submetidas ao laboratório de preparação e análise química, incluindo as amostras da mina e da usina, foi de 480 amostras. Todas as amostras foram preparadas e analisadas no mesmo laboratório, seguindo o mes- mo procedimento de secagem, quarteamento e rebritagem. De cada amostra foram retiradas 3 alíquotas de, aproximadamente, $50 \mathrm{~g}$ cada para análise de ouro, arsênio e enxofre. A média ponderada das 3 alíquotas representou o teor do furo e a média dos teores dos furos ponderada pela respectiva profundidade representou o teor médio do bloco.

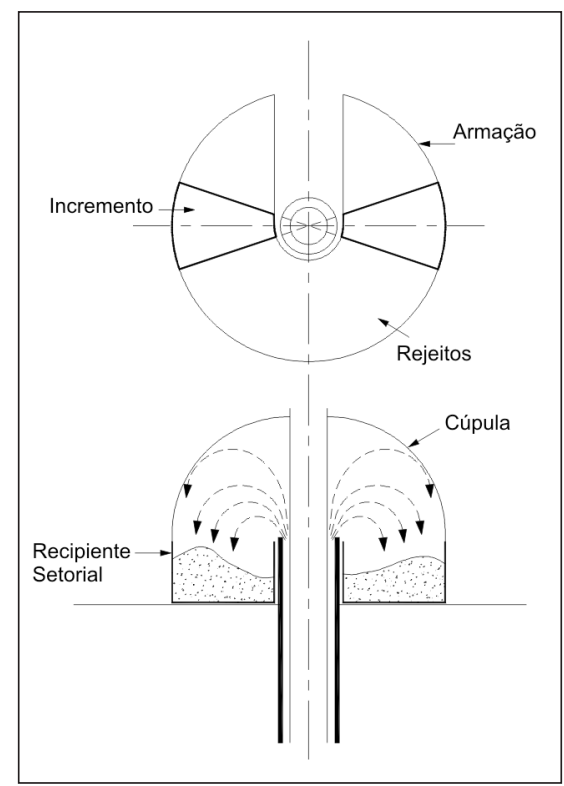

Figura 1 - Amostrador setorial com cúpula para captação de finos.
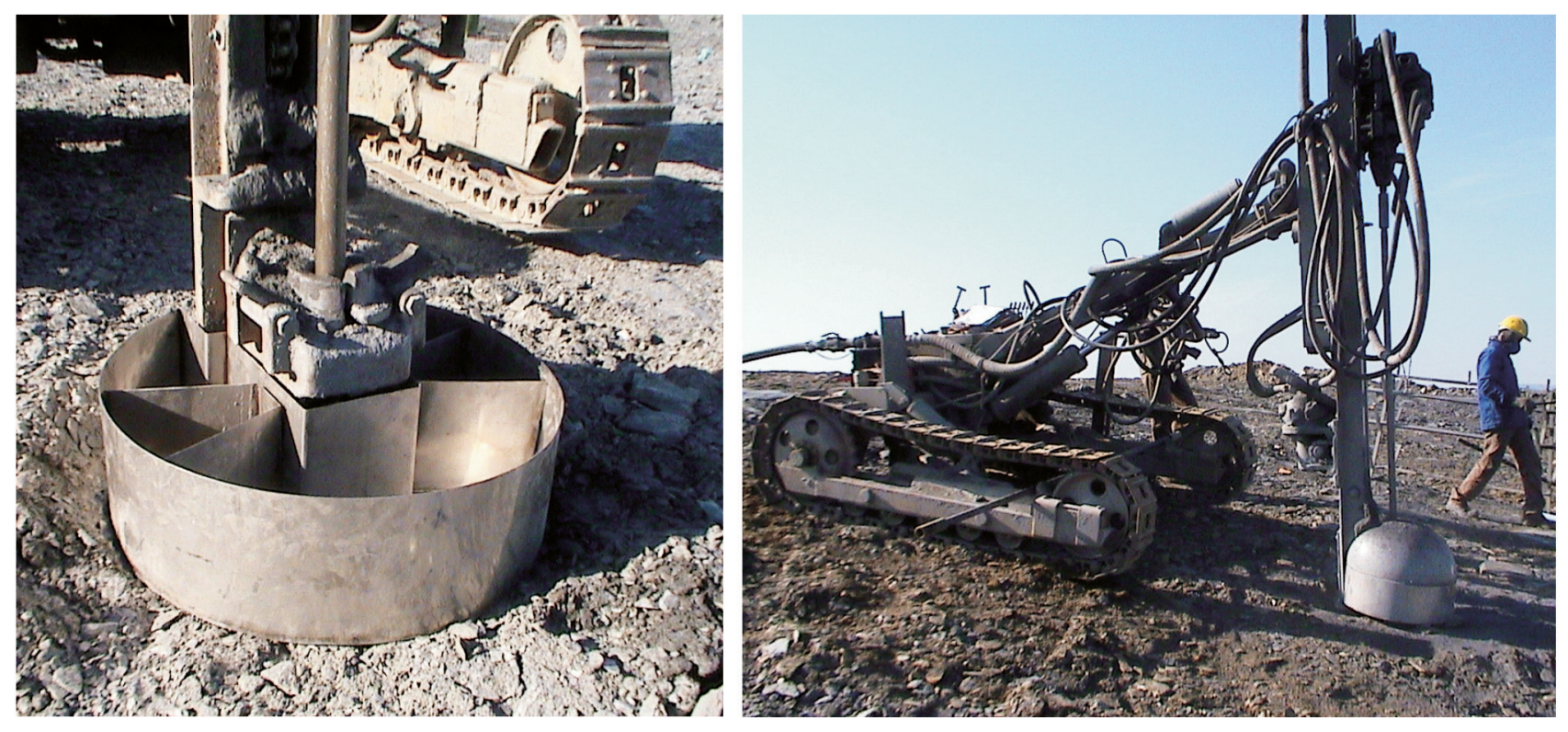

Figura 2 - Amostrador setorial acoplado à lança da perfuratriz. 


\section{Resultados}

A seguir são apresentados os cinco passos da reconciliação pró-ativa, representando as alterações no protocolo de amostragem na mina e na usina, com o objetivo de se reduzirem os erros de estimativa.

1. MINA: substituição da amostragem por pá manual pelo amostrador setorial.

2. MINA: inserção de borracha de vedação sobre a cúpula do amostrador para minimizar perda de finos. USINA: inclusão de amostragem na usina para cálculo de reconciliação.

3. USINA: coleta de um maior número de amostras.

4. MINA: exclusão da borracha de vedação e furação com mais água. USINA: coleta de um maior número de amostras.

5. MINA: reinserção da borracha de vedação e furação sem água. USINA: menor intervalo de tempo entre coleta de amostras.

A Figura 3 mostra a comparação entre os teores estimados pela aplicação do MCF às estimativas do modelo e os teores estimados pelos novos métodos de amostragem na mina e usina.

A Tabela 1 mostra os resultados obtidos para cada bloco e para cada etapa de amostragem. Nota-se que as linhas referentes ao MCF representam o teor de ouro estimado a partir da aplicação do mine call factor às estimativas do modelo da jazida, procedimento comum às práticas de reconciliação reativa.

A análise da Tabela 1 mostra que as práticas de reconciliação reativa não nos permitem prever e/ou controlar os erros de estimativa e, portanto, não são capazes de auxiliar, adequadamente, o planejamento de lavra. A reconciliação próativa, por sua vez, ajudou a compreender alguns dos erros cometidos durante os processos de coleta de amostras, o que tornou possível a alteração de metodologias visando a minimizar esses erros.

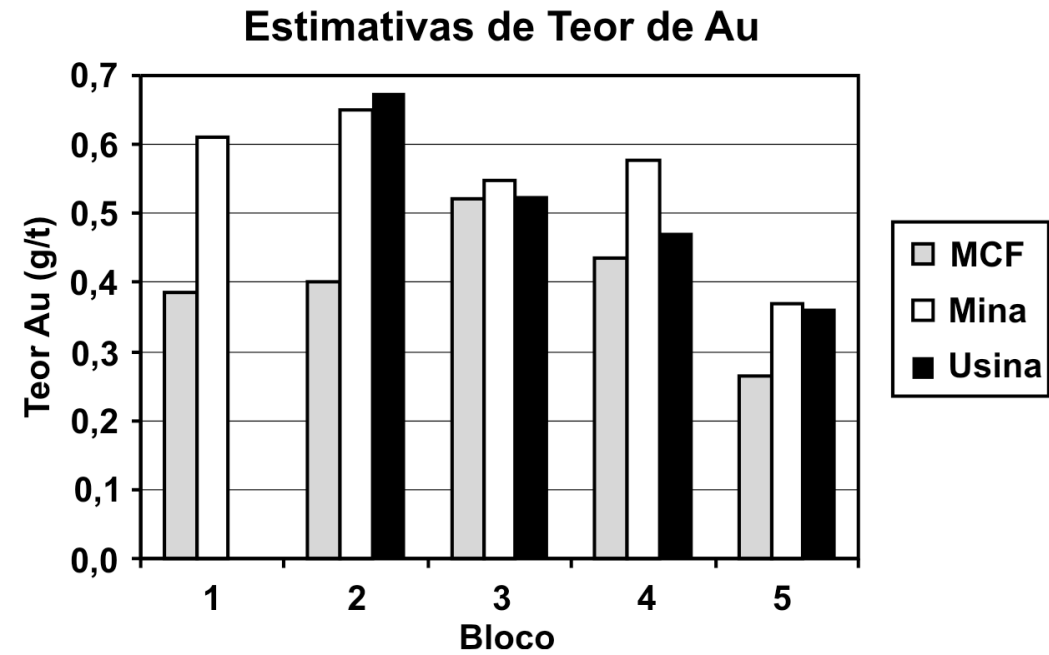

Figura 3 - Teores de ouro estimados pelos diversos métodos.

Tabela 1 - Resultados comparativos entre as práticas de prognosticação e de reconciliação reativa.

\begin{tabular}{|c|c|c|c|c|c|c|}
\hline $\begin{array}{c}\# \\
\text { Bloco }\end{array}$ & $\begin{array}{c}\text { Origem } \\
\text { dos } \\
\text { dados }\end{array}$ & $\begin{array}{c}\text { Teor } \\
\text { médio } \\
\text { de } \mathrm{Au} \\
(\mathrm{g} / \mathrm{t})\end{array}$ & $\begin{array}{c}\text { Média } \\
\text { do erro } \\
\text { m(SE) }\end{array}$ & $\begin{array}{c}\text { Variância } \\
\text { do erro } \\
\mathbf{S}^{2}(\mathrm{SE})\end{array}$ & $\begin{array}{c}\text { Erro de } \\
\text { estimativa de } \\
\text { teor } \\
\text { prognosticação }\end{array}$ & $\begin{array}{c}\text { Erro de } \\
\text { estimativa de } \\
\text { teor } \\
\text { reconciliação }\end{array}$ \\
\hline 1 & $\begin{array}{l}\text { MCF } \\
\text { Mina }\end{array}$ & $\begin{array}{l}0,385 \\
0,612\end{array}$ & * & * & * & * \\
\hline 2 & $\begin{array}{l}\text { MCF } \\
\text { Mina } \\
\text { Usina }\end{array}$ & $\begin{array}{l}0,400 \\
0,649 \\
0,673\end{array}$ & $-0,024$ & 0,054 & $3,50 \%$ & $40,60 \%$ \\
\hline 3 & $\begin{array}{l}\text { MCF } \\
\text { Mina } \\
\text { Usina }\end{array}$ & $\begin{array}{l}0,522 \\
0,548 \\
0,524\end{array}$ & 0,029 & 0,053 & $4,60 \%$ & $0,38 \%$ \\
\hline 4 & $\begin{array}{l}\text { MCF } \\
\text { Mina } \\
\text { Usina }\end{array}$ & $\begin{array}{l}0,436 \\
0,578 \\
0,471\end{array}$ & 0,103 & 0,040 & $22,70 \%$ & $7,40 \%$ \\
\hline 5 & $\begin{array}{l}\text { MCF } \\
\text { Mina } \\
\text { Usina }\end{array}$ & $\begin{array}{l}0,265 \\
0,369 \\
0,363\end{array}$ & $-0,011$ & 0,028 & $1,70 \%$ & $26,90 \%$ \\
\hline
\end{tabular}

* Não há valor de referência, pois não houve amostragem na usina. 
Sabendo que a variância do erro de amostragem representa a precisão ou reprodutibilidade das amostras e que a média do erro de amostragem representa a acurácia ou enviesamento das amostras, a seqüência cronológica mostrou uma melhoria nas estimativas, traduzida pela diminuição da variância do erro de amostragem (de 0,054 para 0,028 ), pela diminuição da média do erro de amostragem (de - 0,024 para $-0,011$ ) e pela diminuição dos erros de estimativa do teor de ouro (de 3,5\% para 1,7\%).

A exceção, não menos importante que a regra, é feita ao bloco 4, que, apesar de exaustivamente amostrado, apresentou erros acima do esperado. A provável causa desse enviesamento foi o aumento, particularmente para esse bloco, da quantidade de água utilizada na perfuração, com o intuito de diminuir a geração e a conseqüente expulsão dos finos. Na realidade, o que ocorreu foi uma "lavagem" dos finos para dentro do furo e, assim, a primeira condição de representatividade de uma amostra não foi satisfeita: a condição de não-enviesamento. As estatísticas, felizmente, confirmam que, sem dados de qualidade, qualquer análise perde o sentido.

\section{Discussão}

Uma amostra é considerada representativa quando as duas seguintes condições forem satisfeitas: a amostra é acurada (não-enviesada) e precisa (suficientemente reproduzível). Como as práticas de reconciliação baseiam-se nos resultados de amostragem, um método de reconciliação deve sempre ter por objetivo aumentar a representatividade das amostras, maximizando, portanto, sua acurácia e precisão. Os resultados das práticas de prognosticação apresentados nesse trabalho mostraram, cronologicamente, o aumento da representatividade das amostras, traduzido por:

1. Maior acurácia: diminuição da média do erro de amostragem, $\mathrm{m}(\mathrm{SE})$.

2. Maior precisão: diminuição da variância do erro de amostragem, $\mathrm{s}^{2}(\mathrm{SE})$.

\section{Conclusões}

Mesmo conhecendo os conceitos da teoria da amostragem, nem sempre se consegue realizar, industrialmente, o teoricamente correto. A amostragem de ouro, em especial, tem suas peculiaridades, principalmente no que diz respeito à segregação entre as partículas e à dificuldade em se reduzir a massa de uma amostra sem causar alterações significativas em seu teor. Todos os problemas com a amostragem de ouro são ampliados quanto menor o teor de ouro, quanto mais marginal o depósito e quanto mais irregular a distribuição do ouro na rocha. O presente trabalho estudou um depósito de ouro de baixo teor, utilizando amostras provenientes do pó de perfuratriz, onde a precisão de amostragem é baixa e, em geral, há enviesamento das amostras.

Portanto, partiu-se da pior situação e procurou-se desenvolver uma metodologia de amostragem que, no mínimo, permitisse conhecer os erros envolvidos nos processos, de modo que os resultados finais pudessem ser usados, conscientemente, nos cálculos de reconciliação. A qualidade desses resultados depende da qualidade e da confiabilidade dos dados de entrada. E, portanto, especial atenção foi dada à geração de dados confiáveis, ou amostras representativas, procurando seguir as regras básicas de seleção de amostras corretas.

Como uma alternativa à reconciliação reativa, foi introduzido o método de reconciliação pró-ativa, ou prognosticação, o qual se baseia no processo iterativo de análise e correção de metodologias de coleta de amostras, com o objetivo de tornar mínima a diferença entre as estimativas e as medições.

Os resultados obtidos mostraram que:

1. Com a diminuição das variâncias dos erros, aumentou-se a precisão da amostragem.

2. Com a diminuição das médias dos erros, $\mathrm{m}(\mathrm{SE})$, aumentou-se a acurácia da amostragem.

3. Com o aumento da precisão e da acurácia da amostragem, aumentou-se a representatividade das amostras e, conseqüentemente, a confiabilidade nos dados de entrada.

Conclui-se, portanto, que a prognosticação pode trazer benefícios significativos a um empreendimento mineiro. É evidente que os erros de amostragem não foram eliminados por completo, mas um passo inicial foi dado e as melhorias foram demonstradas. A aplicação de protocolos capazes de eliminar os erros que podem ser eliminados e minimizar os erros que não podem ser eliminados é uma ferramentas que nos permite criar um modelo cujas estimativas sejam previsões, ou prognósticos, assegurando que os resultados futuros correspondam ao planejamento presente.

\section{Agradecimentos}

À Rio Paracatu Mineração, representada pelos Engenheiros Luis Alberto Alves, Paulo F. Gontijo, Fernanda B. Carneiro, Rodrigo de L. Peroni e Maryse Belanger, pelo Analista de Sistemas Anastácio B. Pains e sua equipe, pelos companheiros de turno da Britagem e por todos os profissionais do Planejamento, Mina, Usina e Hidrometalurgia com quem os autores tiveram a chance de trabalhar. 


\section{Referências bibliográficas}

CRAWFORD, G. D. Reconciliation of reserves: part 2. Pincock Perspectives, n.50, jan/04, p. 1-4, 2004.

FRANÇOIS-BONGARÇON, D., GY, P. Critical aspects in mill and plants: a guide to understanding sampling audits. Journal of the South African IMM, v. 102, n.8, 2002.

GRIGORIEFF, A., COSTA, J.F.C.L., KOPPE, J. O problema de amostragem manual na indústria mineral. $\boldsymbol{R} \boldsymbol{E} \boldsymbol{M}$ :
Revista Escola de Minas, v. 55, n.3, p. 229-233, jul/set, 2002.

GY, P. Sampling for analytical purposes. West Sussex, England: John Wiley \& Sons, , 1998. 172p. (Translated by A.G. Royle.

MORLEY, C. Beyond reconciliation: a proactive approach to using mining data. In: Large Open Pit Mining Conference, $5^{\text {th }}$. Anais... Kalgoorlie, 2003. p. 185192.

PITARD, F. F. Pierre Gy's sampling theory and sampling practice: heterogeneity, sampling correctness, and statistical process control. $2^{\text {nd }}$ ed., Boca Raton: CRC Press, 1993. 488p.

SNOWDEN, V. Comparative 3-D resource modeling approaches at Macraes deposit in New Zealand and their reconciliation with production. In: Applications of Computers in the Mineral Industry. New South Wales: University of Wollongong, 1993. p. 42-45.

Artigo recebido em 09/10/2007 e aprovado em 07/05/2008. 